Philosophical Magazine Letters, Vol. 95, No. 2, pp. 101-109 (2015),

doi: 10.1080/09500839.2015.1013516

\title{
Oriented sulphides induced by electric current in medium carbon steel
}

\author{
X.F. Zhang*, W.J. Lu and R.S. Qin* \\ Department of Materials, Imperial College London, Exhibition Road, London SW7 2AZ, \\ United Kingdom
}

\begin{abstract}
Oriented sulphides parallel to the electric current direction have been experimentally observed when a pulsed electric current is passed through medium carbon steels with $\mathrm{MnS}$ inclusions. Two different configurations of sulphides occur after the application of the electric current: namely, oriented elongated ellipsoidal particles and oriented chain-like particles composed of two or three small spherical sulphides. Theoretical analysis indicates that this phenomenon is associated with minimisation of the electrical resistance of the material.
\end{abstract}

Keywords: pulsed electric current; inclusions; electrical conductivity; steel

*Corresponding authors. Tel.: +44 (0)20 7594 6803; fax: +44 (0)20 7594 6757; e-mail addresses: xfzhangosaka@gmail.com (X.F. Zhang);r.qin@imperial.ac.uk (R.S. Qin) 


\section{Introduction}

Impurities such as inclusions, precipitates and second phases can present in metals and alloys and influence the mechanical properties at high and/or low service temperatures $[1,2]$. These particles also affect the electrical properties of metals and alloys, e.g. by reducing the electrical conductivity. Appling an external field, which can be thermal, electric or magnetic, might affect the configurations of these impurities. These changes in configurations might induce improvement or deterioration of the mechanical and electrical properties. Of these fields, electric fields are especially convenient for practical purposes since their magnitudes, phases, and frequencies can easily be adjusted. When an electric current passes through a conductor, the system free energy contains an additional term $G_{e}$ in comparison with the system without electric current but in the same state (temperature, pressure, and constituents, etc.). The term $G_{e}$ has the following form $[3,4]$ :

$$
G_{e}=-\frac{\mu}{8 \pi} \iint_{V} \frac{\vec{j}(r) \cdot \vec{j}\left(r^{\prime}\right)}{\left|r-r^{\prime}\right|} d r d r^{\prime}
$$

where $\mu$ is the magnetic permeability, $V$ is the volume of the material, and $r$ and $r^{\prime}$ are two different positions in space. $\vec{j}(r)$ is the current density at position $r$. The distribution of electric current density is affected by the spatial configuration of electrical conductivity. If the configuration of impurities in a metal can be changed by an electric current, the electrical conductivity of part or of the whole sample changes. This causes an alternation of current distribution from $\vec{j}_{1}$ to $\vec{j}_{2}$, and hence a change of electric-current-associated free energy $\Delta G_{e}$. $\Delta G_{e}$ can be expressed as [4,5]:

$$
\Delta G_{e}=\frac{\mu}{8 \pi} \iint_{V} \frac{\vec{j}_{1}(r) \vec{j}_{1}\left(r^{\prime}\right)-\vec{j}_{2}(r) \vec{j}_{2}\left(r^{\prime}\right)}{\left|r-r^{\prime}\right|} d^{3} r d^{3} r^{\prime}
$$

where the sub-indices 1 and 2 represent the states before and after microstructural evolution. Normally, numerical calculation can be performed to give the quantitative value of $\Delta G_{e}[3]$, but the calculation is a complex process. In essence, $\Delta G_{e}$ is proportional to a geometric factor $k$, the square of the current density $j^{2}$, and to $\frac{\sigma_{1}-\sigma_{2}}{2 \sigma_{1}+\sigma_{2}}$. Thus the general expression of $\Delta G_{e}$ can be expressed as follows [6]: 


$$
\Delta G_{e} \infty \frac{\sigma_{1}-\sigma_{2}}{2 \sigma_{1}+\sigma_{2}} k j^{2}
$$

where $\sigma_{1}$ and $\sigma_{2}$ are the electric conductivities of the matrix and impurity, respectively. The geometric factor $k$ is positive, and is associated with the dimensions of the matrix and impurity. The experimental and calculated results show that the electric current promotes a structural evolution in the material towards the state with overall lower electric resistance $[4,7,8]$. The configuration (e.g. orientation and/or morphology) of the impurities in the metal matrix will affect the geometric factor $k$ and produce an arrangement toward a certain direction so that the electrical resistance along the electrical current direction is at minimum. It can be seen from Figure 1 that the geometric morphology of each dark phase with high electrical resistivity in the low-resistivity bright matrix can be divided into three types, namely a randomly oriented ellipsoidal phase (Figure 1a-1), a randomly oriented small spherical phase (Figure 1b-1), and a randomly oriented large spherical phase (Figure 1c-1). The spatial configurations of these phases affect the total electrical resistance of the material significantly. In order to minimise the electrical resistance, the configurations of the phases in Figure1a-1, 1b-1 and 1c-1 might be arranged towards the electrical current direction, that is, the structure might evolve as illustrated in Figure 1 (e.g. from Figure 1a-1 to 1a-2 to 1a-3; from Figure $1 \mathrm{~b}-1$ to $1 \mathrm{~b}-2$ to $1 \mathrm{~b}-3$; and from Figure $1 \mathrm{c}-1$ to $1 \mathrm{c}-2$ to $1 \mathrm{c}-3)$. Here, it should be noted that the large spherical particles in Figure 1c-1 might be modified to ellipsoidal particles of Figure 1c-2 owing to the alteration of the interfacial energy during the electric current treatment. In addition to the thermodynamic aspects of these, the formation of the aligned microstructure in Figure 1 is also encouraged by enhanced diffusion along the electric current direction in the alloy (i.e. kinetics aspects). The possibilities for enhancing the diffusion by pulsed electric currents include an increase in the diffusion pre-exponential factor and/or a reduction in the diffusion activation energy [9]. Previously it has been reported that elongated $\mathrm{SnPb}$ eutectic grains and growth of lamellae occurred along the electrode directions [10] and that recrystallized nuclei in a cold-rolled silicon steel preferred to form along the current direction [11]. Furthermore, electric-current-induced oriented nanotwins formation with low electrical resistivity in $\mathrm{Cu}-\mathrm{Zn}$ alloy [6] also suggests that the orientation of impurities may be affected by the application of electric current. 

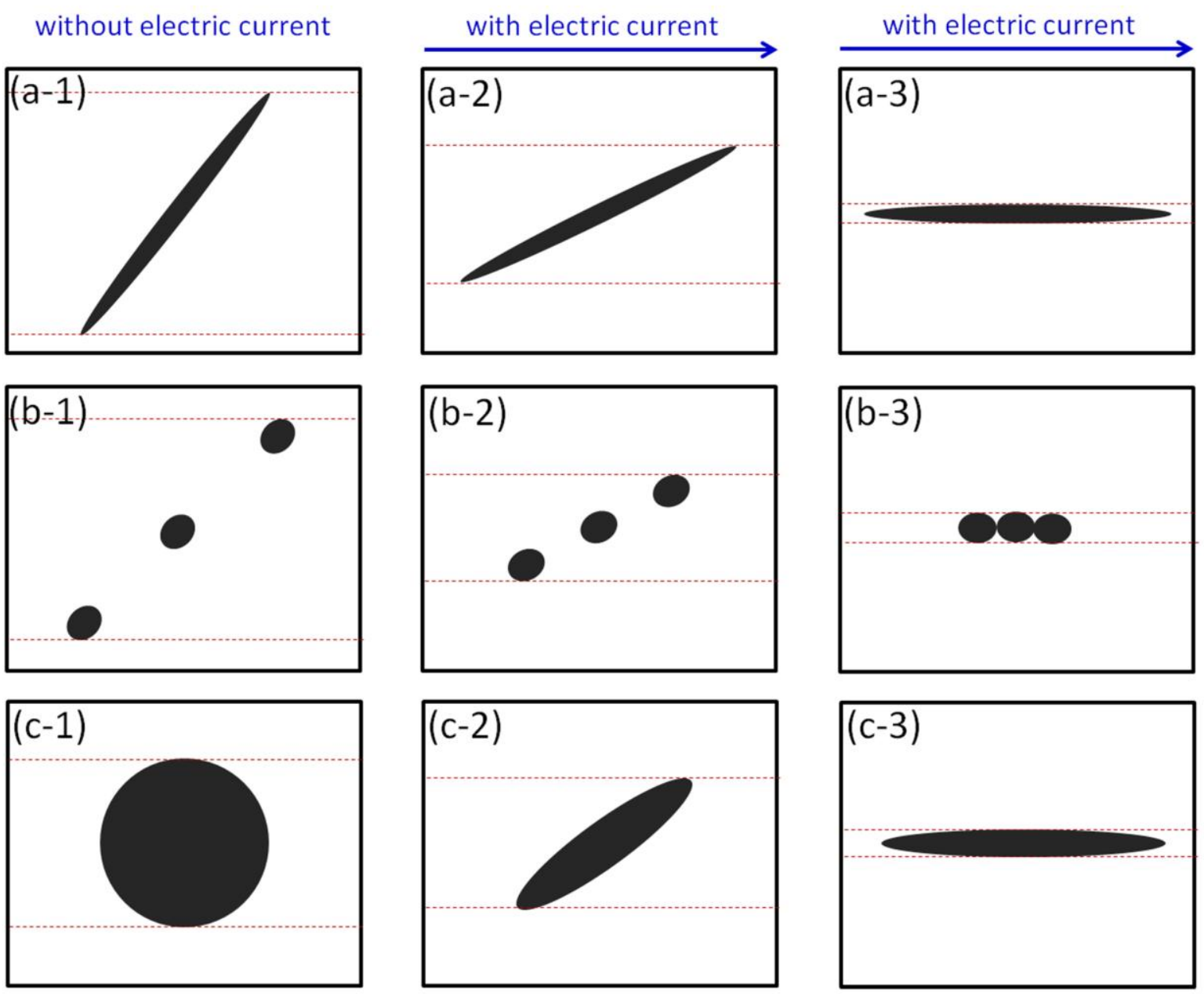

Figure 1 Schematic configuration of MnS particle before and after application of an electric current. The dark particle has a low electric conductivity while the bright matrix has a high electric conductivity. The volume fraction of each particle remains constant. The left section (Figure 1a-1, 1b-1, and 1c-1) illustrates the configuration of the particle before application of the electric current. The middle and right sections (Figure 1a-2, 1a-3; 1b-2, 1b-3; 1c-2, 1c-3) show the rearrangement of the particle after the electric current. The electrical resistance of the material, initially high, is therefore lowered on account of the orientation of the particle in the electrical current direction (e.g. the direction of the arrow).

Concerning impurities, inclusion of a stable particle at an elevated temperature is of particular interest. $\mathrm{MnS}$ inclusions in structural steels are known for their beneficial role during machining $[12,13]$. However, they have, in comparison to other inclusions such as oxides and silicates, the property of being softer than the steel matrix [14,15]. Thus a high thermal expansion coefficient between $\mathrm{MnS}$ and the matrix results in a large stress concentrator 
where a fatigue crack is initiated [13-15]. As shown in Figure 2, a fatigue crack is initiated at a MnS particle when the load axis is perpendicular to the sulphide (Figure 2a and b), while no crack emanates from the sulphide when the load axis is parallel to the particle (Figure $2 \mathrm{c}$ and d) [13-15]. These findings suggest that fatigue anisotropy can be attained if an oriented inclusion can be induced by an electric current. Although fatigue anisotropy is not always expected to occur, forged steel generally suffers from strong fatigue anisotropy owing to the modified morphology of soft inclusions by forging or rolling at elevated working temperatures. Before the process of hot-rolling, if the $\mathrm{MnS}$ inclusion can be arranged to face a certain direction, one can change the arranged particle (elongated or chain-like type in Figure 1a-3/1c-3 and 1b-3) into a globular type sulphide when the rolling direction is perpendicular to the oriented inclusion. By keeping $\mathrm{MnS}$ globular, fatigue isotropy can be markedly improved. Here, $\mathrm{MnS}$ is only an example, and in general other particles whose configurations are influenced by electric fields can be used to fabricate and design novel microstructures. In this letter, a possible configuration of oriented $\mathrm{MnS}$ particles in medium carbon steel will be described, providing insight into the effect of electric currents on orientation of inclusions.

\section{Experimental details}

The sample used was medium carbon steel containing $\mathrm{MnS}$ inclusions. The steel was placed in a graphite crucible and heated to a molten state at approximately $1520{ }^{\circ} \mathrm{C}$. The $\mathrm{MnS}$ inclusions with melting point of $1610{ }^{\circ} \mathrm{C}$ remain solid in the molten steel. In order to apply the electric pulse, a pair of steel bars was submerged into the liquid steel to act as electrodes. To determine the effect of the electric current, two sets of experiments were carried out, with and without the current. The experimental procedures were otherwise identical. The frequency of the applied pulses was $1 \mathrm{~Hz}$ and the duration of each pulse was $60 \mu \mathrm{s}$. The density of the pulsed electric current was $1.8 \times 10^{6} \mathrm{~A} / \mathrm{m}^{2}$ and the total treatment time was 6 minutes. A longitudinal section was observed to detect the configuration of the inclusions. The furnace-cooled samples were longitudinally sectioned and polished for metallographic examination. The configuration of the inclusions was initially examined by optical microscopy to confirm the overall distribution of the particles. The configuration of the inclusions before and after the electric current was examined by transmission electron microscopy (TEM). For these observation, the specimens were mechanically polished to a 
thickness of $30 \mu \mathrm{m}$ and punched to discs $3 \mathrm{~mm}$ in diameter by a brass cutter. The thin-film specimens were electrically polished using a solution $(30 \mathrm{ml}$ perchloric acid and $270 \mathrm{ml}$ acetic acid) at $15^{\circ} \mathrm{C}$ and observed by a TEM operated at an acceleration voltage of $200 \mathrm{kV}$.

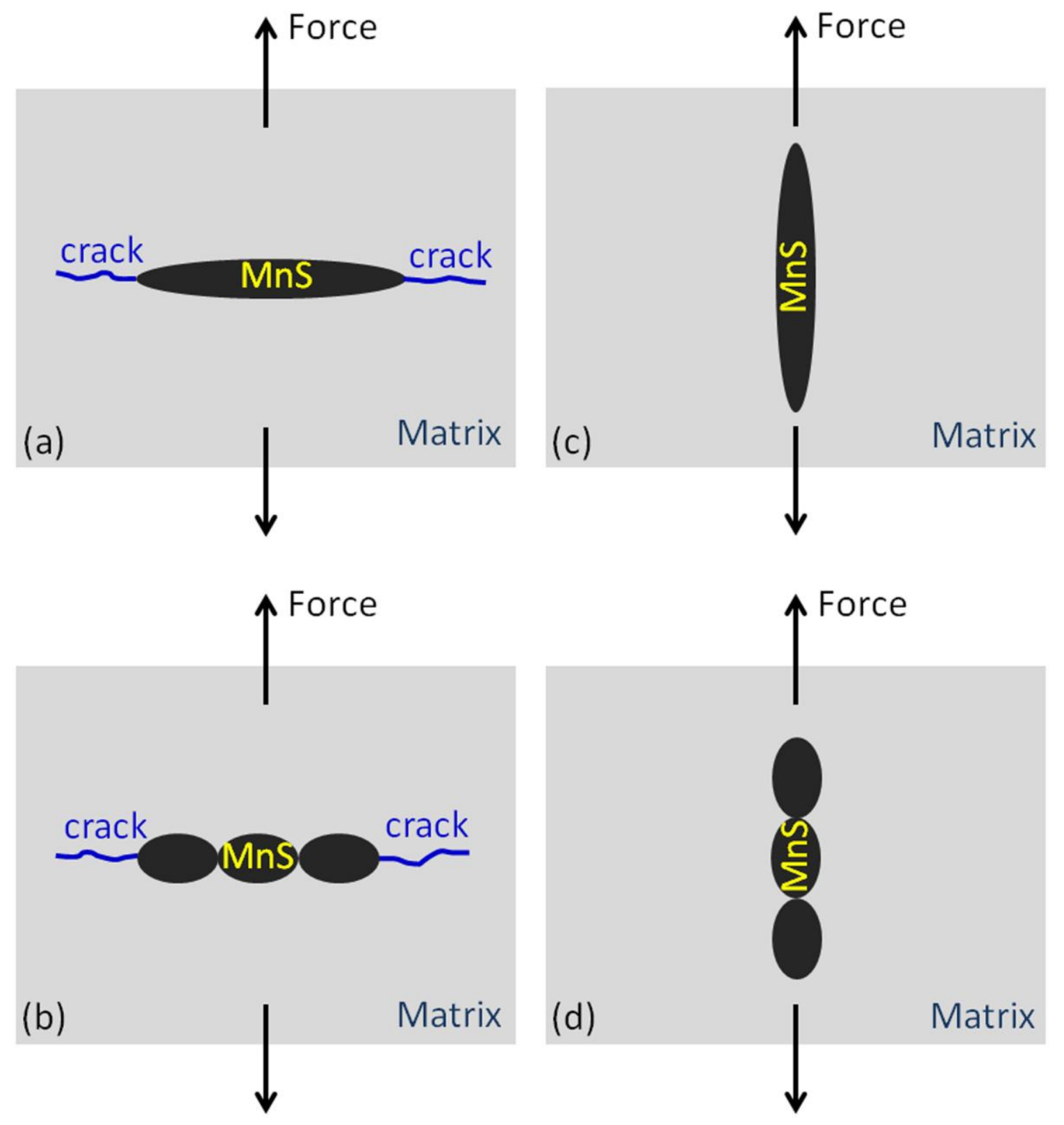

Figure 2 Fatigue crack initiation from different configurations of the $\mathrm{MnS}$ particles. (a) Cracks are along single ellipsoidal $\mathrm{MnS}$ inclusion when the load axis is perpendicular to the sulphide; (b) Cracks emanates from the chain-like type $\mathrm{MnS}$ inclusions when the load axis is perpendicular to the sulphides; (c) and (d) no crack emanates from the sulphide when the load axis is parallel to the particles. 


\section{Results and discussion}

The distribution of $\mathrm{MnS}$ inclusions in the steel before applying the electric current is as shown in Figure $3 \mathrm{a}$ and $\mathrm{b}$. It can be clearly seen that under lower magnification dark $\mathrm{MnS}$ inclusions are dispersed in the steel matrix (Figure 3a). Most of the inclusions are approximately spherical or angular with a diameter of $8 \mu \mathrm{m}$ (corresponding to Figure 1a-1, b1 and c-1), as shown in Figure $3 b$ under high magnification. Each particle is isolated, and the minimum distance between them is approximately $14.5 \mu \mathrm{m}$. By observing the configurations of 40 particles in Figure 3a, it can be found that they are randomly dispersed in the matrix. After the application of the electric current the MnS particles show a tendency to be aligned in the direction in which the current was applied (Figure 3c). There are about 16 angular/spherical particles and 20 ellipsoidal particles. Most of the spherical particles are composed of 2 or 3 small spherical sulphides. These small sulphides are connected to each other, forming a chain-like large particles as shown in Figure 1b-3. In case of the ellipsoidal particles, the direction of their long axis is parallel to the electric current direction as indicated in Figure 1a-3 or Figure 1c-3. The configurations of Figure 1a-2/1c-2 and 1b-2 can also be found in small number in the pulsed steel in Figure 3c. It can be clearly seen from Figure $3 \mathrm{~d}$ with high magnification that the length of the major axis ranges from $8 \mu \mathrm{m}$ to 22.4 $\mu \mathrm{m}$. It seems that some large spherical particles are modified to ellipsoidal particles on account of a modified interfacial energy (e.g. see evolution from Figure 1c-1 to 1c-2). The different interfacial energy between spherical and ellipsoidal particles will contribute to their morphological change. As indicated in Eqs.(2) and (3), the higher $\Delta G_{e}$ in the pulsed steel might result in a decrease in interfacial energy to minimize the system free energy.

In order to confirm the orientation and morphology of the MnS inclusions (especially for the particles less than $3 \mu \mathrm{m}$ in size), the bright-field TEM images of the untreated and pulsed steel were obtained. It will be seen that there are significant difference between these (Figure $4 \mathrm{a}$ and $\mathrm{b}$ ) in that two types of configurations of $\mathrm{MnS}$ particles are aligned along the electric current direction. In case of the steel before the electric current (Figure 4a), MnS inclusions were observed in the pearlitic steel (bright $\alpha$-ferrite and dark cementite). The MnS particles in the untreated steel exhibited a fine equiaxed grain structure a few hundreds nanometers in size. This MnS had a cubic crystal structure by electron diffraction analysis (Figure 4c). By calculations based on the diffraction pattern in Figure 4c, its lattice parameter is $5.17 \AA$ and 
its zone axis is along [ $1 \overline{1} 14]$. Therefore, the particle can be assumed to be $\alpha-\mathrm{MnS}$ with a facecentered-cubic crystal structure [12]. Remarkably, two different types of MnS inclusions are formed after the application of the electric current (Figure 4b). One is MnS particles elongated along the electric current direction (corresponding to Figure 1a-3/1c-3). Another is that two or three small particles are aggregated together to form a chain-like arrangement (corresponding to Figure 1b-3/1b-2). These small particles are connected to each other and form a large particle, but the boundaries between them are not visible. The direction of the major axis of the newly constructed particle is also parallel to the electric current direction. The length of the major axis of the particles ranges from $1.4 \mu \mathrm{m}$ to $2.5 \mu \mathrm{m}$.

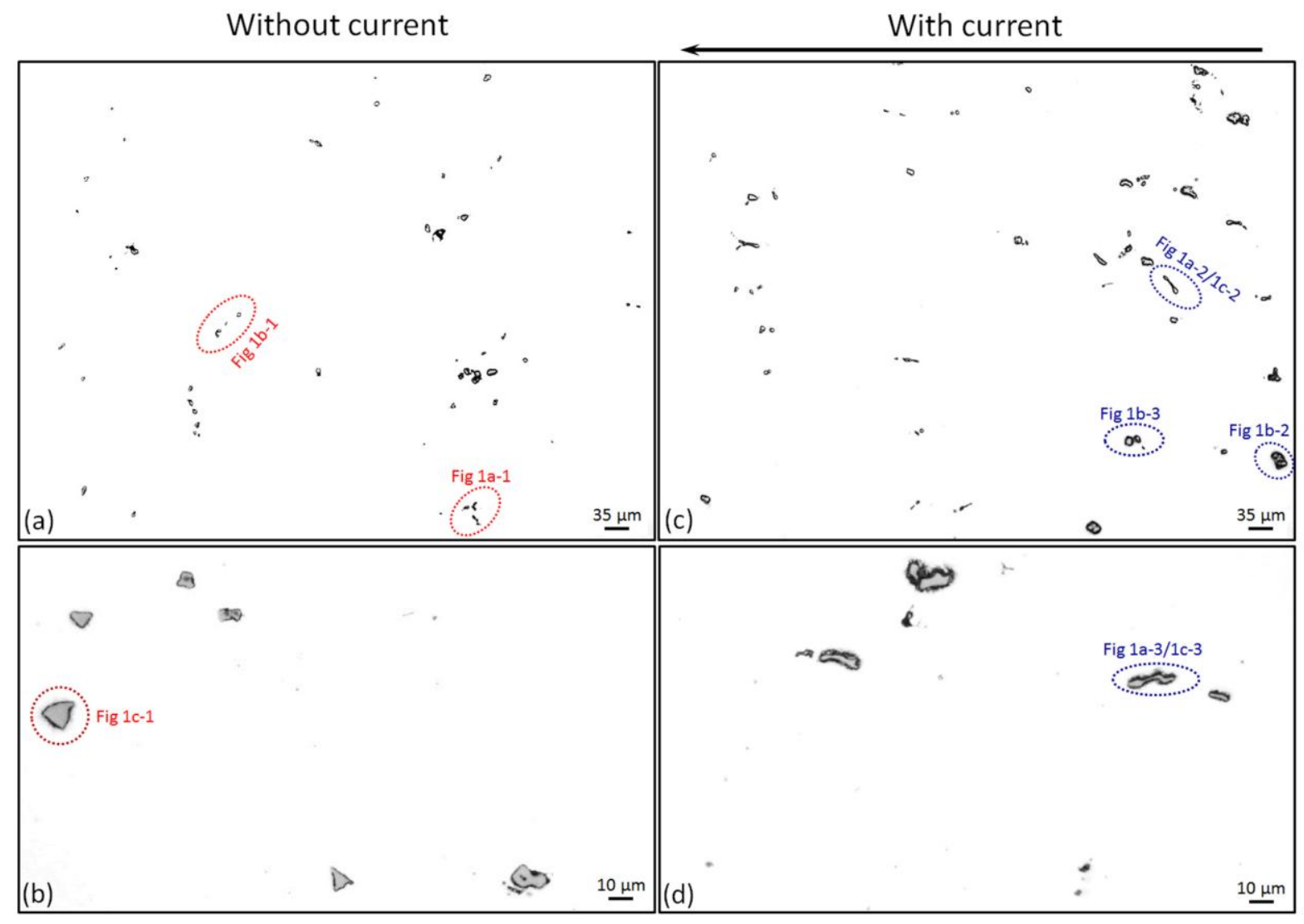

Figure 3 Optical images (a, b) of untreated as-solidified steel and (c, d) of pulsed steel. The direction of the arrow represents the electric current direction. 

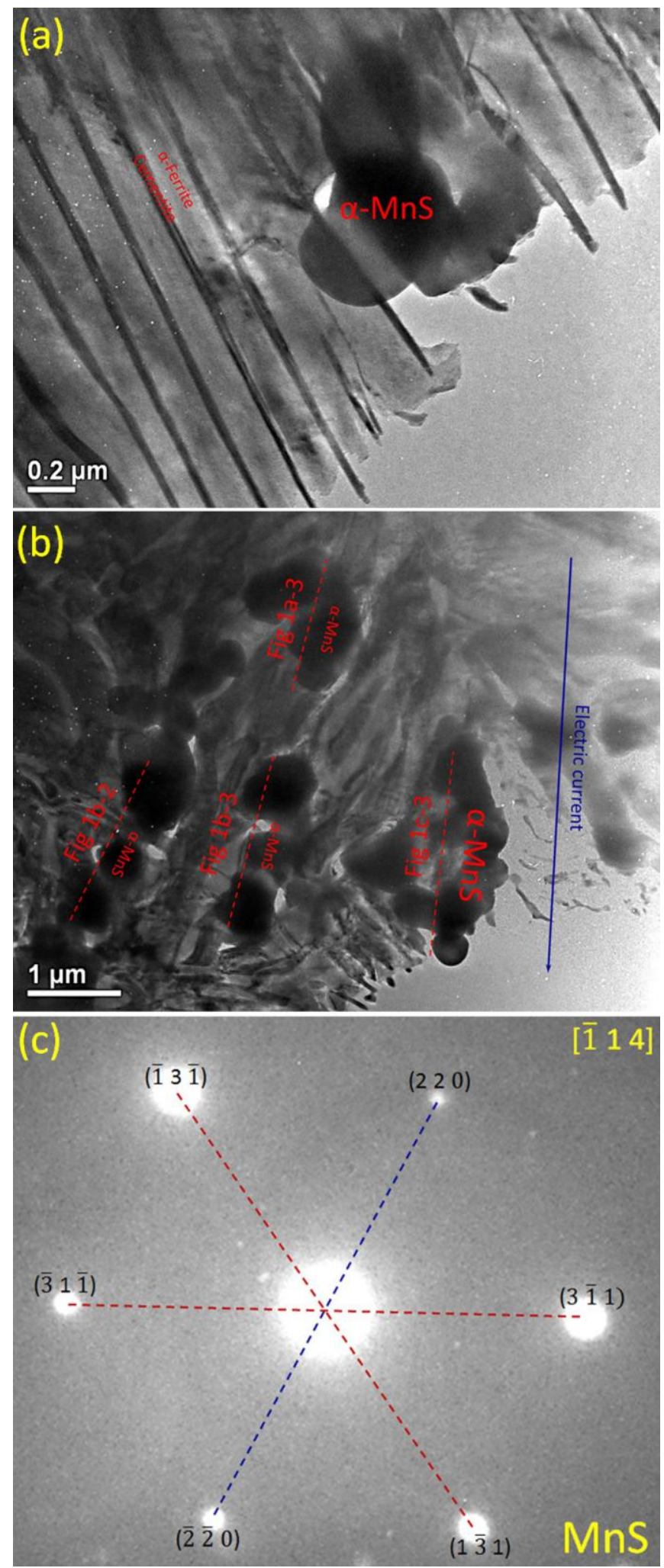

Figure 4 TEM images (a) of untreated as-solidified steel and (b) pulsed steel; (c) the corresponding selected-area electron diffraction pattern was taken on the dark particle showing a cubic $\alpha-\mathrm{MnS}$ along [ $1 \overline{1} 14]$ zone axis. 
The above results show that both the large and small sulphides ( $>8 \mu \mathrm{m}$ in optical images and $<3 \mu \mathrm{m}$ in TEM images) had been re-configured during application of the electric current, the direction of the major axis of the newly configured sulphide being parallel to the electric current direction. This is consistent with the speculation of Figure 1 based on Eqs. (2)and (3). Here, the electrical conductivity of the $\mathrm{MnS}$ is $10^{3}$ smaller than that of the steel [5]. In order to reduce the electric resistance of the system, $\mathrm{MnS}$ inclusions are re-configured leaving the system with a high electrical conductivity. Owing to the formation of oriented $\mathrm{MnS}$ inclusions, a fatigue anisotropy of the material is introduced. Fatigue anisotropy is generally considered to be detrimental to materials. However, forged steel generally suffers from strong fatigue anisotropy arising from a morphology modified by soft inclusions during forging or rolling operations at elevated working temperatures. For the pulsed steel, the ellipsoidal or chain-like shapes of the oriented $\mathrm{MnS}$ particles can be rolled to be globular when the rolling direction is perpendicular to the oriented inclusion. In simple hot-rolling or forging, it is difficult to obtain a globular shaped particle, while this configuration can be easily realized in the hot-rolled pulsed steel. In general, a globular type $\mathrm{MnS}$ is desirable, because this configuration is conducive to the fatigue isotropy. On the other hand, the formation of precipitates or second phase particles is also very beneficial for improving the mechanical and electrical properties of the material. For example, electric current pulsing induced the formation of oriented nanotwins with a high electrical conductivity and greatly enhanced the mechanical and electrical properties of a $\mathrm{Cu}-\mathrm{Zn}$ alloy [6]. Furthermore, electric current pulsing may also lead to a corresponding change in the texture of materials [16-18]. This has great significance for the applications of Mg alloys, $\mathrm{Al}$ alloys, and electrical silicon steel. In general, a fundamental understanding of particle configurations modified by electric currents can help in the fabrication and design of novel microstructures.

\section{Conclusions}

The effect of pulsed electric currents can minimise the electrical resistance of a material. In particular in this work oriented sulphides parallel to the electric current direction have been configured in medium carbon steel. Optical and TEM observations confirm that such configurations can be produced in both large sulphides (> $8 \mu \mathrm{m}$ in optical images) and small sulphides $(<3 \mu \mathrm{m}$ in TEM images). Such a phenomenon provides a special and effective approach to fabricating and designing novel microstructures. 


\section{Acknowledgements}

The work was financially supported by EPSRC (No. EP/J011460/1), TATA Steel and the Royal Academy of Engineering at United Kingdom. The authors are grateful to Professor Kenneth C. Mills at Imperial College London and Mr Andrew Smith and Mr Michael McDonald at TATA Steel Teesside Technology Centre for fruitful discussions. We are grateful to TATA Steel Europe for the provision of steel samples used in the experiments.

\section{Reference}

[1] C. Punckt, M. Bölscher, H.H. Rotermund, A.S. Mikhailov, L. Organ, N. Budiansky, J.R. Scully and J.L. Hudson, Science 305 (2004) p.1133.

[2] M.P. Ryan, D.E. Williams, R.J. Chater, B.M. Hutton and D.S. McPhail, Nature 415 (2002) p.770.

[3] X.F. Zhang and R.S. Qin, Appl. Phys. Lett. 104 (2014) p.114106.

[4] R.S. Qin, E.I. Samuel and A. Bhowmik, J. Mater. Sci. 46 (2011) p.2838.

[5] X.F. Zhang, W.J. Lu and R.S. Qin, Scr. Mater. 69 (2013) p.453.

[6] X.L. Wang, Y.B. Wang, Y.M. Wang, B.Q. Wang and J.D. Guo, Appl. Phys. Lett. 91 (2007) p.163112.

[7] Y. Dolinsky and T. Elperin, Phys. Rev. B 50 (1994) p.52.

[8] R.S. Qin, A. Rahnama, W.J. Lu, X.F. Zhang and B. Elliott-Bowman, Mater. Sci. Technol. 30 (2014) p.1040.

[9] Y.Z. Zhou, W. Zhang, J.D. Guo and G.H.He, Philos. Mag. Lett.84 (2004) p.341.

[10] J.M. Li, S.L. Li, J. Li and H.T. Lin, Scr. Metal. Mater. 31 (1994) 1691.

[11] W.B. Dai, X.L. Wang, H.M. Zhao and X. Zhao, Mater. Trans. 53 (2012) 229.

[12] N. Matsui and K. Watari, ISIJ Int. 46 (2006) p.1720. 
[13] C. Temmel, B. Karlsson and N.G. Ingesten, Fatigue Fract. Engng. Mater. Struct. 31 (2008) p.466.

[14] A. Melander, Int. J. Fatigue 19 (1997) p.13.

[15] T.J. Baker, K.B. Gove and J.A. Charles, Met. Technol. 3 (1976) p.183.

[16]L. Guan, G.Y. Tang, P.K. Chu and Y.B. Jiang, J. Mater. Res. 24 (2009) p.3674.

[17]C.Y. Liu, Y.J. Hu, Y.S. Liu, H.W. Tseng, T.S. Huang, C.T. Liu, Y.C. Chuang and S.L. Cheng, Acta Mater. 61 (2013) p.5713.

[18] G.L. Hu, Y.H. Zhu, C.H. Shek and G.Y. Tang, J. Mater. Res. 26 (2011) p.917. 